\title{
NMR in Copper-Oxide Metals
}

\author{
C. M. Varma \\ AT\&T Bell Laboratories, Murray Hill, NJ 07974
}

\begin{abstract}
The anomalous part of the NMR relaxation rate of copper nuclei in the normal state of copperoxide metals is calculated using the orbital magnetic parts of the fluctuations derived in a recent theory to explain the long wavelength transport anomalies. Oxygen and Yttrium reside on lattice sites at which the anomalous contribution is absent at all hole densities. The frequency, momentum dependence, and the form-factor of the fluctuations is predicted, which is verifiable by inelastic neutron scattering experiments.
\end{abstract}


Of all the various anomalies in the normal state of copper-oxide metals, ${ }^{1}$ the most extraordinary are the nuclear magnetic relaxation ${ }^{2}$ rates $\mathrm{T}_{1}^{-1}$. These rates probe local fluctuations and are a very detailed and stringent test of microscopic theories. The anomalies are essentially identical in the best studied materials, $\mathrm{YBa}_{2} \mathrm{Cu}_{3} \mathrm{O}_{6+\mathrm{x}}$ with $\mathrm{x} \approx 0.9\left(123 \mathrm{O}_{6.9}\right)$ and $\mathrm{La}_{2-\delta} \mathrm{Sr}_{\delta} \mathrm{CuO}_{4}$ with $\delta \approx 0.15$ i.e. near the composition for the highest $\mathrm{T}_{\mathrm{c}}$. The local magnetic fluctuations in a metal are usually of the form: $\operatorname{Im} \chi(\omega) \sim \frac{\omega \mathrm{N}(0)}{\mathrm{E}_{\mathrm{F}}}$ leading to $\mathrm{T}_{1}^{-1} \sim \mathrm{T}$. The oxygen $\mathrm{T}_{1}^{-1}$ in the $\mathrm{Cu}-\mathrm{O}$ metals are consistent with this behavior, ${ }^{3}$ as is that of $\mathrm{Y}$ nuclei in (123). ${ }^{4}$ The Cu-nuclear relaxation rate, on the other hand, is ${ }^{5}$ to a first approximation, ${ }^{\mathrm{Cu}} \mathrm{T}_{1}^{-1} \sim \mathrm{a}+\mathrm{bT}$ with $\mathrm{a} \approx 6 \mathrm{~T}_{\mathrm{c}}$. The (nearly) constant part in ${ }^{C u} \mathrm{~T}_{1}^{-1}$ suggests that the local magnetic fluctuations in $\mathrm{Cu}$ have a scale-invariant form at low energies: $\chi^{\prime \prime}(\omega) \sim \omega / \mathrm{T},{ }^{6}$ unlike Fermi-liquids and as if the metals lie near a $\mathrm{T}=0$ critical point. This is remarkable enough. But it is even more remarkable that the local electronic fluctuations on the oxygen do not share the fluctuations on $\mathrm{Cu}$ even though the electronic wavefunctions on the two ions are well hybridized.

One idea discussed extensively ${ }^{7}$ to explain the observations is that the materials are near an antiferromagnetic instability of the $\mathrm{Cu}$ magnetic moments with a magnetic correlation length $\xi^{-1} \sim$ T. Since the oxygen ions sit half way between the coppers, they do not see the antiferromagnetic fluctuations. Direct measurements ${ }^{8}$ of $\chi^{\prime \prime}(q, \omega)$ by inelastic neutron scattering show this idea to be untenable. In $123 \mathrm{O}_{6.9}$ the magnetic correlation length is ${ }^{8}$ less than a lattice constant at low energies and temperature independent. In $\mathrm{La}_{1.85} \mathrm{Sr}_{.15} \mathrm{CuO}_{4}$, magnetic correlations at low energies are indeed observed. ${ }^{9}$ But the correlations are at an incommensurate wave-vector so that the fluctuations do not cancel on the oxygen ions. Calculations ${ }^{10}$ of $\mathrm{T}_{1}^{-1}$ using the measured $\chi^{\prime \prime}(\mathrm{q}, \omega)$ give a temperature dependence which is quite different from the experiments for both ${ }^{0} \mathrm{~T}_{1}^{-1}$ and ${ }^{\mathrm{Cu}} \mathrm{T}_{1}^{-1}$. Moreover, $\left({ }^{0} \mathrm{~T}_{1} \mathrm{TK}\right)^{-1} \approx(1.4 \pm 0.2) \mathrm{sec}^{-1} \mathrm{deg}^{-1}$ for all measured temperatures in the normal phase for $123 \mathrm{O}_{6+\mathrm{x}}$ for several different $\mathrm{x}$ and for $\mathrm{La}_{1.85} \mathrm{Sr}_{.15} \mathrm{CuO}_{4} \cdot{ }^{11}$ Here $\mathrm{K}$ is the measured oxygen Knight shift. This is in agreement with the value obtained ${ }^{12}$ from the conventional Korringa Law, in terms of the deduced hyperfine coupling constants. The temperature independence of this quantity, 
as well as its near invariability from one compound to another is inexplicable by AFM correlations whose q-vector and magnitude change from compound to compound and with $\mathrm{x}$. Some more robust symmetry is called for so that the fluctuations seem by $\mathrm{Cu}$ nuclei are absent at the oxygen sites. Clearly, something more subtle is at work.

Recently a model for copper-oxide metals ${ }^{13,14}$ has been systematically investigated. ${ }^{14} \mathrm{~A} \mathrm{~T}=0$ critical point has been discovered at a unique composition $\mathrm{x}=\mathrm{x}_{\mathrm{c}}$, at which the fluctuations lead to marginal Fermi-liquid self energy: $\operatorname{Im} \Sigma(\omega) \sim \max (\omega, \mathrm{T}) \operatorname{sgn} \omega$, and long wavelength transport properties in agreement with experiment. Here these fluctuations are applied to calculate features of $\mathrm{T}_{1}^{-1}$.

For reasons discussed elsewhere, ${ }^{13,14}$ the model includes dynamical degrees of freedom both on $\mathrm{Cu}$ and on $\mathrm{O}$ ions and includes long-range interactions besides a strong short-range repulsion on $\mathrm{Cu}$ ions to exclude the $\mathrm{Cu}^{3+}$ state. For long-range interaction energies comparable to or larger than the $\mathrm{Cu}-\mathrm{O}$ bonding - antibonding splitting, the model has a critical point at $\mathrm{T}=0$, and hole density $\mathrm{x}=\mathrm{x}_{\mathrm{C}}$. Near this point (intracell $\mathrm{Cu}-\mathrm{O}$ ) current fluctuations have a propagator ${ }^{14}$

$$
\mathrm{D}(\mathbf{q}, \omega)=\left(1 / \mathrm{W}_{\mathrm{s}}\right)\left(\left\{\mathrm{i} \omega / \gamma+\ln \left(\omega_{\mathrm{c}} / \gamma\right)\right\}^{-1}+\mathrm{Aq}^{2}+\mathrm{G}(\mathrm{x}, \mathrm{T})\right)^{-1}
$$

Here $\mathrm{W}_{\mathrm{s}}$ is the spectral weight of the fluctuations and $\gamma(\omega, \mathrm{T}, \mathrm{G})$, the damping of this unconserved mode is $\approx \max \left(|\omega|, \mathrm{T}, \mathrm{G}, \tau_{0}^{-1}\right) . \quad \tau_{0}^{-1}$ is the order of the elastic rate for single particle scattering from impurities, obtainable, from resistivity measurements. The "mass" of the collective mode, $\mathrm{G}(\mathrm{x}, \mathrm{T})=\mathrm{g}_{0}\left|\mathrm{x}-\mathrm{x}_{\mathrm{c}}\right|+\mathrm{T} / \mathrm{T}_{0}$, where $\mathrm{g}_{0}$ is $\mathrm{O}(1)$ and $\mathrm{T}_{0}$ is the order of the bandwidth.

We now consider the orbital magnetic contribution of these current modes to the magnetic fluctuations: $\chi(\mathrm{q}, \omega)$. First, consider the local fluctuations around any particular ion with the $\mathrm{Cu}-$ O metal treated in the one-electron approximation. The local hyperfine Hamiltonian for a nuclear spin $\mathbf{I}$ chosen to be at the origin is

$$
\mathrm{H}_{\mathrm{hf}}=\mathbf{I} \cdot \mathbf{M}=\mathbf{I} \cdot \sum_{\mathrm{kq}} \mathbf{M}_{\mathrm{kq}}(0) \mathrm{c}_{\mathrm{k}+\mathrm{q} / 2}^{+} \mathrm{c}_{\mathrm{k}-\mathrm{q} / 2},
$$


where $\mathbf{M}_{\mathrm{k}, \mathrm{q}}(0)$ is the matrix element of the magnetization at the origin between Bloch waves: ${ }^{15}$

$$
\mid \mathrm{k}>=\mathrm{e}^{\mathrm{ik} \cdot \mathbf{r}} \mathrm{u}_{\mathbf{k}}(\mathbf{r})
$$

The contributions to $\mathrm{M}$ relevant to us are

$$
\mathbf{M}_{\mathrm{orb}}=\mathrm{i} \hbar \frac{\mathbf{L}}{\mathrm{r}^{3}}=\mathrm{i} \hbar \frac{\mathbf{r} \times \nabla}{\mathrm{r}^{3}}
$$

Consider the matrix element

$$
\left\langle\mathbf{k}+\frac{\mathbf{q}}{2}\left|\mathbf{M}_{\text {orb }}\right| \mathbf{k}-\mathbf{q} / 2\right\rangle=\hbar\left\langle\mathrm{e}^{-\mathbf{i q} \cdot \mathbf{r}} \mathrm{u}_{\mathbf{k}+\mathbf{q} / 2}^{*}(\mathrm{r})\left(\frac{\mathbf{k} \times \mathbf{r}}{\mathrm{r}^{3}}+\mathrm{i} \frac{\mathbf{r} \times \nabla}{\mathrm{r}^{3}}\right) \mathrm{u}_{\mathbf{k}-\mathbf{q} / 2}(\mathrm{r})\right\rangle .
$$

The Bloch-functions $u_{\mathbf{k}}(r)$ are in general very complicated. We will content ourselves with general properties of (5) remembering that the relaxation rate sums over all $\mathbf{k}$ and $\mathbf{q}$. To estimate (5) consider $u_{k}$ at some symmetry points where we know its properties, for example the non-bonding point $\mathbf{k}=(0,0)$. At this point $\mathrm{u}_{0}(\mathbf{r})$ can be written as

$$
\frac{1}{\sqrt{\mathrm{N}}} \sum_{\mathrm{i}, \alpha} \mathrm{a}_{\mathrm{i}, \alpha}(0) \phi\left(\mathbf{r}-\mathbf{R}_{\mathrm{i}, \alpha}\right),
$$

where i sums over the cells and $\alpha$ the atoms in the cell: $\phi\left(\mathbf{r}-\mathrm{R}_{\mathrm{i}, \alpha}\right)$ has the symmetry of the $\mathrm{d}_{\mathrm{x}^{2}-\mathrm{y}^{2}}$ orbitals at the $\mathrm{Cu}$-site and has the symmetry of the $\mathrm{p}_{\mathrm{x}}$ and $\mathrm{p}_{\mathrm{y}}$ orbitals, appropriately, at the two oxygen sites per unit cell. The phase factors $\mathrm{a}_{\mathrm{i}, \alpha}(0)$ are \pm 1 , phased to produce the non-bonding configuration at $\mathbf{k}=0$. We can verify by "k.p perturbation" to determine the phase factors $\mathrm{a}_{\mathrm{i}, \alpha}(\mathbf{k})$ for $\mathbf{k}$ about this point that the qualitative results remain the same at arbitrary points in the zone. So we can use (6) to find the qualitative properties of (5).

Inserting (6) in (5), we use the rapid fall-off of $\phi\left(\mathbf{r}-\mathbf{R}_{\mathrm{i} \alpha}\right)$ with $\left|\mathbf{r}-\mathrm{R}_{\mathrm{i} \alpha}\right|$ to approximate (6) by a sum of two terms: (a) $(i, \alpha)$ at the origin (i.e. the position of the nucleus whose relaxation rate is being evaluated) on both $\mathrm{u}(\mathbf{r})$ factors in (5); (b) (i, $\alpha)$ at the origin in one $\mathrm{u}(\mathbf{r})$ and $(\mathrm{i}, \alpha)=$ nearest neighbors of the atom at the origin and vice-versa. In higher order terms the sums over (i, $\alpha$ ) may be replaced by an integral over space. This is then just the fluctuating electromagnetic field at 
the origin due to long wavelength orbital currents. Such effects have been evaluated ${ }^{16}$ and formed to contribute $\mathrm{O}\left(10^{-4}\right)$ to the relaxation rates compared to the on-site relaxation terms (say, from contact hyperfine interactions) and may be ignored.

The local orbital fluctuation term (a) in (6) is zero at both $\mathrm{Cu}$ and oxygen if as in $\mathrm{Cu}-\mathrm{O}$ metals non degenerate orbitals reside at these sites. Consider now the next term (b). The second ${ }^{17}$ term in (5) yields a contribution linear in q given by

$$
\left\langle\mathbf{k}+\frac{\mathbf{q}}{2}\left|\mathbf{M}_{\mathrm{orb}}\right| \mathbf{k}-\frac{\mathbf{q}}{2}\right\rangle \simeq \hbar \sum_{\mathrm{n}} \mathrm{iq} \cdot\left\langle\mathbf{r} \phi\left(\mathbf{r}-\mathbf{R}_{\mathrm{n}}\right) \frac{\mathbf{L}}{\mathrm{r}^{3}} \phi(\mathbf{r})\right\rangle,
$$

where $\mathrm{n}$ sums over the nearest neighbors. Take a $\mathrm{Cu}$-site at the origin; then $\phi(\mathrm{r})$ has $\mathrm{d}_{\mathrm{x}^{2}-\mathrm{y}^{2}}$ symmetry so that $\mathbf{L}_{\mathrm{z}} \phi(\mathrm{r}) \sim \mathrm{d}_{\mathrm{xy}} \cdot \phi\left(\mathrm{r}-\mathbf{R}_{\mathrm{n}}\right)$ have $\mathrm{p}_{\mathrm{x}}$ or $\mathrm{p}_{\mathrm{y}}$ symmetries so that $\sum_{\mathrm{n}} \mathbf{q} \cdot \mathbf{r} \phi\left(\mathbf{r}-\mathbf{R}_{\mathrm{n}}\right)$ for a general $\mathbf{q}$ also has a component with $\mathrm{d}_{\mathrm{xy}}$ symmetry. Finite matrix element of $\mathbf{L}_{\mathrm{z}}$ therefore exist. The construction of a $\mathrm{d}_{\mathrm{xy}}$ symmetry around a $\mathrm{Cu}$ ion by appropriately phasing the oxygen $\mathrm{p}_{\mathrm{x}}$ and $\mathrm{p}_{\mathrm{y}}$ orbitals is illustrated in Fig. 1. Semi-classically, orbital relaxation requires a current fluctuation circulating around the nuclei. This is provided by the loop formed by the four oxygens around a given $\mathrm{Cu}$. These produce magnetization fluctuations in the z-direction which contribute to the nuclear relaxation rate $\mathrm{T}_{1 \perp}^{-1}$ with field applied in the $\mathrm{Cu}-\mathrm{O}$ planes.

The same mechanism also contributes to ${ }^{\mathrm{Cu}} \mathrm{T}_{1 \|}^{-1}$ with field applied normal to the $\mathrm{Cu}-\mathrm{O}$ plane. Since $\mathbf{L}_{\mathrm{x}, \mathrm{y}} \mathrm{d}_{\mathrm{x}^{2}-\mathrm{y}^{2}} \sim \mathrm{d}_{\mathrm{xz}}, \mathrm{d}_{\mathrm{yz}}$ this occurs through the relative phasing of the oxygen $\mathrm{p}_{\mathrm{x}, \mathrm{y}}$ orbitals in the plane and the $\mathrm{p}_{\mathrm{z}}$ orbital of the apical oxygens with a $\mathbf{q}$ in the plane and in general with the apical oxygens mutually out of phase.

Since $\mathrm{L}_{\mathrm{z}} \mathrm{p}_{\mathrm{x}} \sim \mathrm{p}_{\mathrm{y}}$, etc, orbital relaxation of oxygen nuclei requires a fluctuation of $\mathrm{p}_{\mathrm{x}, \mathrm{y}}$ form to be created by phasing the neighbors. For the magnetic fluctuation at the oxygen nuclei, consider (6) with oxygen sites at the origin. Oxygen is linearly coordinated by copper ions, and no contribution of $\mathrm{O}(\mathrm{q})$ exist from the nearest neighbor terms, because $\sum_{\mathrm{n}} \mathbf{q} \cdot \mathbf{r} \phi\left(\mathrm{r}-\mathrm{R}_{\mathrm{n}}\right)$ cannot be phased to yield a p-symmetry at the oxygen at the origin. This is true to any order in q. Semiclassically, no simple closed loop current fluctuation can be created around oxygen unlike the case of copper illustrated 
in Fig. (1). Similarly, $\mathrm{Y}$ is an s-state ion sitting between $\mathrm{Cu}-\mathrm{O}$ planes and no contributions of the type we are considering occur for it.

Magnetic fluctuations, which because of the symmetry of the lattice, contributing only to relaxation of $\mathrm{Cu}$-nuclear spins therefore do exist. We now consider their frequency and temperature dependence. We use the fact that the operators in (4) project on to operators on $\mathrm{Cu}$ and O-orbitals which participate in the current fluctuations of Eq. (3). The magnetization fluctuations then couple to the current fluctuations through a factor proportional to $\mathbf{q}$, as in Eq. (7). We therefore have an anomalous contribution to the relaxation at $\mathrm{Cu}$ nuclei:

$$
\mathrm{T}_{1 \mathrm{an}, \mathrm{i}}^{-1}=(\mathrm{T} / \omega) \sum_{\mathbf{q}}\left\langle\mathbf{M}_{\mathbf{q}} \mathbf{M}_{-\mathbf{q}}\right\rangle_{\omega}=(\mathrm{T} / \omega) \sum_{\mathbf{q}} \alpha_{\mathrm{i}}^{2} \mathbf{q}^{2} \mathrm{D}(\mathbf{q}, \omega)
$$

Here $\alpha_{\mathrm{i}}=\alpha_{\perp}$ or $\alpha_{\|}$, depending on the direction of the magnetic field. The $\alpha$ 's are undetermined numerical factors which depend, besides the couplings in Eq. (3) on details of the structure and chemistry. In (8) q is two-dimensional; the magnetization correlations between the planes is purely from the very small long-range electro-magnetic effects, and assumed smaller than the temperature.

Now we note that near $\mathrm{x}=\mathrm{x}_{\mathrm{c}}$ (ignoring $\log \mathrm{T}$ corrections)

$$
\operatorname{Lim}(\omega \rightarrow 0)(T / \omega) \sum_{\mathbf{q}} \mathrm{q}^{2} \operatorname{ImD}(\mathrm{q}, \omega) \approx \frac{\mathrm{T}}{\mathrm{W}_{\mathrm{s}}}\left[\frac{1}{\gamma_{0}(\mathrm{~T})}+\mathrm{O}\left(1 / \mathrm{E}_{\mathrm{F}}\right)\right]
$$

Since $\gamma_{0}(\mathrm{~T})=\tau_{0}^{-1}+\lambda \mathrm{T}$ at $\mathrm{G}=0$, a scale-invariant contribution to the local fluctuation on $\mathrm{Cu}$ indeed arises in the pure limit, $\lambda \mathrm{T} \gg \tau_{0}^{-1}$.

We write the ordinary contribution to nuclear relaxation rate of a nucleus $n$ with the field applied in the direction $\mathrm{i}$ as

$$
{ }^{\mathrm{n}} \mathrm{T}_{1, \mathrm{i}}^{-1}=\lim _{\omega \rightarrow 0} \sum_{\mathrm{q}, \overline{\mathrm{i}}}\left|{ }^{\mathrm{n}} \mathrm{F}_{\overline{\mathrm{i}}}\left(\mathrm{q}_{\mathrm{i}}\right)\right|^{2} \chi_{0}^{\prime \prime}(\mathrm{q}, \omega) / \mu_{\mathrm{B}}^{2}
$$

In (10), ${ }^{n} F_{i}(q)$ include the measured hyperfine constants as well as the lattice form factors, as given, for instance, in Ref. (18). $\overline{\mathrm{i}}$ are orthogonal to i. To this we supplement for $\mathrm{Cu}$ the anomalous 
contribution coming from (9),

$$
{ }^{\mathrm{Cu}} \mathrm{T}_{1 \text { an,i }}^{-1}=\frac{\alpha_{\mathrm{i}}^{2}}{\mathrm{~W}_{\mathrm{s}}} \frac{\mathrm{T}}{\gamma_{0}(\mathrm{~T})}
$$

Equation (10) has been evaluated extensively, with $\chi_{0}^{\prime \prime}$ calculated in various approximations ${ }^{19,12}$ and with the form of $\chi_{0}^{\prime \prime}(q, \omega)$ deduced from experiments ${ }^{9,10}$ for $\mathrm{La}_{1.86} \mathrm{Sr}_{.14} \mathrm{CuO}_{4}$ which show incommensurate antiferromagnetic correlations. ${ }^{10}$ We defer the role of such correlations, which in any case are of an ignorable magnitude in $123 \mathrm{O}_{6.93}$, and write (10) in the Fermi-liquid form, so that

$$
{ }^{\mathrm{Cu}_{1, \mathrm{i}}} \mathrm{T}_{1}^{-1}=\left.\left.\frac{\chi_{0}}{\mu_{\mathrm{B}}^{2} \mathrm{E}_{\mathrm{F}}} \mathrm{T}\right|^{\mathrm{Cu}} \mathrm{A}_{\overline{\mathrm{i}}}\right|^{2}+\frac{\alpha_{\mathrm{i}}^{2}}{\mathrm{~W}_{\mathrm{s}}} \frac{\mathrm{T}}{\gamma_{0}(\mathrm{~T})}
$$

and

$$
{ }^{0} \mathrm{~T}_{1, \mathrm{i}}^{-1}=\left.\left.\left(\frac{\chi_{0}}{\mu_{\mathrm{B}}^{2} \mathrm{E}_{\mathrm{F}}}\right) \mathrm{T}\right|^{0} \mathrm{~A}_{\overline{\mathrm{i}}}\right|^{2}
$$

where in ${ }^{\mathrm{n}} \mathrm{A}_{\mathrm{i}}$, we have absorbed the hyperfine constants, form factors and other factors after scaling out a factor $\left(\chi_{0} / \mu_{\mathrm{B}}^{2} \mathrm{E}_{\mathrm{F}}\right)$. Note also that $\alpha_{\mathrm{i}}$ has the same dimensions as $\mathrm{A}_{\mathrm{i}}$.

We now quantitatively compare (12) with the experimental results. We first note that $\lim q \rightarrow 0$, $\omega \rightarrow 0 \operatorname{Re} \mathrm{q}^{2} \mathrm{D}(\mathrm{q}, \omega)=\alpha^{2} / \mathrm{W}_{\mathrm{s}}$, so that for $\alpha^{2} \ll 1$, this provides a negligible contribution to the uniform magnetic susceptibility and the Knight shift. So the hyperfine constants deduced from the measured Knight shift should be used only in the usual (Fermi-liquid-like) contributions to $\mathrm{T}_{1}^{-1}$. At high temperatures, $\mathrm{T} \gg \tau_{0}^{-1},(12)$ behaves as $\mathrm{a}+\mathrm{bT}$; the constant part is replaced by the term proportional to $\mathrm{T} \tau_{0}$ for $\mathrm{T} \gg \tau_{0}^{-1}$. If three dimensional coupling of the anomalous magnetization fluctuations were significant, it would also produce a term proportional to $\mathrm{T}$.

In Fig. (2) we compare the calculated result for ${ }^{\mathrm{Cu}} \mathrm{T}_{1, \perp}^{-1}$ from Eq. (12) with field in the plane with the experimental results in $\left(123 \mathrm{O}_{6.93}\right)$. We deduce $\left.\left.\frac{\chi_{0}}{\mu_{\mathrm{B}}^{2} \mathrm{E}_{\mathrm{F}}}\right|^{\mathrm{Cu}} \mathrm{A}_{\perp}\right|^{2}$ by scaling up the measured $\left(\mathrm{T}_{1} \mathrm{~T}\right)^{-1}$ of oxygen by the ratio of the appropriate measured hyperfine coupling constants. We can estimate $\gamma_{0}(\mathrm{~T})$ from the measured resistivity. The fit in Fig. (2) is with $\lambda \approx 1$ and $\tau_{0}^{-1} \approx 25 \mathrm{~K}$, similar to what one deduces from the measured resistivity of $\left(1230_{6.93}\right)$. This leaves the undetermined parameter, $\alpha_{\perp}^{2} / \mathrm{W}_{\mathrm{s}}$, to fit the data. The fit in Fig. (2) is with $\alpha_{\perp}^{2} / \mathrm{W}_{\mathrm{s}} \approx 3.2(\mathrm{msec})^{-1}$, which with $\mathrm{W}_{\mathrm{s}} \approx \mathrm{E}_{\mathrm{F}} \approx 10^{4} \mathrm{~K}$ implies $\mid \alpha_{\perp} /{ }^{\mathrm{Cu}} \mathrm{A}_{\perp} / 2 \approx 6 \times 10^{-2}$. 
We also compare the ${ }^{\mathrm{Cu}_{\mathrm{T}}} \mathrm{T}_{1}^{-1}$ measured by NQR in a single crystal of $\mathrm{La}_{1.86} \mathrm{Sr}_{14} \mathrm{CuO}_{4}$. The only significant difference in parameters needed to fit the data is a larger value $\tau_{0}^{-1} \approx 10^{2} \mathrm{~K}$. This is consistent with the fact that the extrapolated residual resistivity of optimum (124) crystals is generally higher than that of optimum (123) crystals. The fit in Fig. (2) uses $\alpha_{\perp}^{2} / \mathrm{W}_{\mathrm{s}} \approx 2.8(\mathrm{msec})^{-1}$.

To see if $\alpha_{\perp}^{2}$ is reasonable, we compare it to a hypothetical situation where $d_{x^{2}-y^{2}}$ and $d_{x y}$ orbitals on $\mathrm{Cu}$ are degenerate, so that a local orbital as well as a dipolar fluctuation contribution to $\mathrm{T}_{1}^{-1}$ exists with field in the plane. The ratio $\left|\mathrm{A}_{\mathrm{orb}} / \mathrm{A}_{\perp}\right|^{2}$ in this case (where both relaxation rates are $\sim \mathrm{T}$ ) can be easily calculated ${ }^{20}$ and is $\approx 20$. We have two reduction factors in $\alpha^{2}$ compared to $\mathrm{A}_{\text {orb }}^{2}$, that due to $\left\langle\phi_{\mathrm{i}}^{2}\right\rangle \sim \mathrm{x}$, and more importantly, because the distance to the neighboring oxygen p-orbitals is involved. A reduction factor of $\mathrm{O}\left(10^{-2}\right)$ is therefore not unreasonable.

In general $\left(\alpha_{\perp} / \alpha_{\|}\right)^{2}>1$ is expected, the details depending on structure. Experimentally this ratio is about 2.6 in $\mathrm{La}_{1.85} \mathrm{Sr}_{.15} \mathrm{CuO}_{4}$ and about 4 in $\mathrm{YBa}_{2} \mathrm{Cu}_{3} \mathrm{O}_{6.9}$. This is in the right direction from the consideration here since the former is a single layer and the latter a double layer compound. Measurements of anisotropy in other compounds appear not to have been made. We would predict that this ratio rises in compounds with larger number of layers. Any numerical estimates do not appear feasible.

At compositions away from the ideal, i.e. $\mathrm{g}_{0}\left|\mathrm{x}-\mathrm{x}_{\mathrm{c}}\right|>>\mathrm{T} / \mathrm{T}_{0}$, the fluctuation modes of eq. (1) have a gap. This has the same effect in $\mathrm{T}_{1}^{-1}$ as an enhanced $\tau_{0}^{-1}$. This is qualitatively consistent with the experimental results that there is a smoother variation of $\mathrm{T}_{1}^{-1}$ from low temperatures to high away from the ideal composition. It is worth noting also that a finite $\mathrm{G}$ also leads to a cross-over at temperatures of $\mathrm{O}(\mathrm{G})$ from a lower effective mass to a higher effective mass. This should be observable in thermodynamic properties: both specific heat and magnetic susceptibility as indeed it is. ${ }^{21}$

As has been discussed before, ${ }^{12,19}$ the ${ }^{\mathrm{O}} \mathrm{T}_{1}^{-1}$ in $\left(123 \mathrm{O}_{7}\right)$ is consistent with (13) and given quantitatively in terms of the measured $\chi_{0}$ and hyperfine constants. This is true in the present theory at all $\mathrm{x}$. For ${ }^{0} \mathrm{~T}_{1}^{-1}$ in $\mathrm{La}_{1.85} \mathrm{Sr}_{.15} \mathrm{CuO}_{4}$ to be consistent with the neutron scattering results, the 
absolute magnitude of the q-dependent part of $\chi^{\prime \prime}(\mathrm{q}, \omega)$ at low $\omega$ should be about a factor of 3 less than used in Ref. (10) or the deduced (hyperfine constants) ${ }^{2}$ smaller by a similar factor.

In contrast to $\mathrm{T}_{1}^{-1}$ which is related to fluctuations integrated over $\mathbf{q}, \mathrm{T}_{2}^{-1}$ of $\mathrm{Cu}$, as derived by Pennington and Slichter ${ }^{2}$ is dependent primarily to fluctuation at $\mathrm{q} \approx \pi / \mathrm{R}_{\mathrm{Cu}-\mathrm{Cu}}$. So antiferromagnetic correlations, however weak are picked up in $\mathrm{T}_{2}^{-1}$. Berthier et al. ${ }^{11}$ find using the neutron scattering results as a function of temperature in $123 \mathrm{O}_{6.7}$ and in $123 \mathrm{O}_{6.93}$ that the measured correlation length of only about two lattice constants in the former and one or less in the latter accounts for the measured $\mathrm{T}_{2}^{-1}(\mathrm{~T})$. Similar conclusions have been arrived at by Walstedt for $\mathrm{La}_{1.86} \mathrm{Sr}_{.14} \mathrm{CuO}_{4}$.

¿From Eqs. (1), (4) and (7) one can deduce that the orbital magnetic fluctuations contribute

$$
\operatorname{Im} \chi_{\operatorname{orb}}(\mathrm{q}, \omega) \simeq \mu_{\mathrm{B}}^{2}\left(\frac{\mathrm{a}_{0}}{\mathrm{a}}\right)^{6}\left(\mathrm{a}^{2} \mathrm{q}^{2}\right) \operatorname{Im} \mathrm{D}(\mathrm{q}, \omega)
$$

to the total magnetic fluctuations, $\operatorname{Im} \chi(\mathrm{q}, \omega)$, with a form factor such that they are seen only at the $\mathrm{Cu}$ (or symmetry equivalent) sites and absent at the oxygen (or symmetry equivalent) sites. Here $\mathrm{a} \approx 1.9 \AA$ is the nearest neighbor $\mathrm{Cu}-\mathrm{O}$ distance, and $\mathrm{a}_{0}$ is of the order of the atomic radius. To this should be added the usual spin-fluctuation contributions. Should there be significant (but not singular) AFM correlations, for example due to nesting, the Fermi-liquid contributions to Eqs. (12) and (13) can easily be modified to include their effect.

A contribution, smooth in both q and $\omega$ extending up to high energies, as in Eq. (14) is indeed observed. ${ }^{22}$ To test Eq. (14) in detail requires measurements up to high frequencies at various temperatures. The only measurement reported ${ }^{22}$ up to high frequencies has been done only at $\mathrm{T}=17 \mathrm{~K}$ in $\mathrm{La}_{1.85} \mathrm{Sr}_{.15} \mathrm{CuO}_{4}$. Fig. (3) of Ref. (22) gives the frequency dependence of the integrated absorption as very slowly decreasing to $\sim 0.2 \mathrm{eV}$ below a weak peak at $\sim 20 \mathrm{meV}$. This is quite consistent with Eq. (14). Measurements at other temperatures in that compound and up to high frequencies and various temperatures in $123 \mathrm{O}_{6.9}$ are urged. Especially crucial are measurements of the fluctuation spectrum in several Brillouin zones to deduce separately the fluctuations centered 
on $\mathrm{Cu}$ alone and on $\mathrm{O}$ alone The former should see (14) while the latter should be the usual Fermiliquid form, i.e. $\approx \mathrm{N}(0) \omega / \mathrm{q} V_{\mathrm{F}}$ for $\omega \lesssim q \mathrm{~V}_{\mathrm{F}}$ and $\approx 0$ beyond. These are hard experiments, but they should resolve the principal problem in the field.

I am pleased to acknowledge very helpful discussions with A. Sengupta, G. Kotliar, P. B. Littlewood and Q. Si about this work, and extensive discussions on NMR with R. E. Walstedt, H. Alloul, C. Berthier and C. Hammel and on neutron scattering with G. Aeppli. 


\section{References}

1. For a review, see B. Batlogg in High Temperature Superconductivity, ed. by K. Bedell et al., Addison Wesley, New York (1989).

2. For review, see C. H. Pennington and C. P. Slichter in Physical Properties of High Temperature Superconductors II, D. M. Ginsburg, editor, World Scientific, Singapore (1989).

3. P. C. Hammel et al., Phys. Rev. Lett. 63, 1992 (1989)

4. H. Alloul, T. Ohno and P. Mendels, Phys. Rev. Lett. 63, 1700 (1989)

5. R. E. Walstedt et al., Phy. Rev. B 38, 9299 (1988).

6. C. M. Varma et al., Phys. Rev. Lett. 63, 1996 (1989).

7. B. S. Shastry, Phys. Rev. Lett. 63, 1288 (1989).

8. J. Rossat-Mignod et al., Physica 192 B, 109 (1993); J. M. Tranquada et al. Phys. Rev. B46, 5561 (1992); H. A. Mook et al., Phys. Rev. Lett. 70, 3490 (1993); L. P. Regnault et al., Physica C 235, 59 (1994); B. Keimer et al., (preprint).

9. T. E. Mason et al., Phys. Rev. Lett. 71, 919 (1993).

10. R. E. Walstedt, B. S. Shastry and S.-W. Cheong, Phys. Rev. Lett. 72, 3610 (1994). It is also worth noting that the result reported (T. Imai, Phys. Rev. Lett. 70, 1002 (1993)) that at high temperatures $\mathrm{La}_{1.85} \mathrm{Sr}_{.15} \mathrm{CuO}_{4}$ has the same ${ }^{\mathrm{Cu}} \mathrm{T}_{1}^{-1}$ as $\mathrm{La}_{2} \mathrm{SrCuO}_{4}$ has been modified by more recent measurements (M. Matsumra et al., J. Phys. Soc. Japan 65, 699 (1996)).

11. C. Berthier et al., Physica C 185-9, 1141 (1991); Physica Scripta T49, 131 (1993) and private communication; R. Walstedt (unpublished).

12. P. B. Littlewood et al., Phys. Rev. B 48, 487 (1993). 
13. C. M. Varma, S. Schmitt-Rink and E. Abrahams, Solid State Comm. 62, 681 (1987).

14. C. M. Varma, Phys. Rev. Letters. 75, 898 (1995) and Preprint (1996), submitted to Physical Review and available on http://www.lanl.gov as cond-mat/9607105. In this preprint, some important modifications of the results in the PRL are given. In particular, a damping mechanism of the collective mode due to particle-hole scattering near the Fermi-surface leads to the form of $\gamma(\omega, T)$ in Eq. (1) which is more important than Landau damping for all momenta.

15. In the tight-binding approximation the phase factors for electronic wave-functions is given only at the lattice points. The results derived below depend on varying the phase of the wave-functions between lattice points. Therefore Bloch waves must be used for the electronic wave-functions.

16. P. A. Lee and N. Nagaosa, Phys. Rev. 43, 1223 (1991).

17. For general k's there is also a contribution from the first term in (5) with properties similar to those evaluated here. Since we are unable to evaluate any of the matrix elements quantitatively and the aim is only to show the feasibility of the idea and fit to experiment with reasonable parameters, we do not discuss these.

18. (a) F. Mila and T. M. Rice, Phys. Rev. B 40, 11382 (1989); (b) A. J. Millis and H. Monien, Phys. Rev. B 45, 3059 (1992).

19. Q. Si, Y. Zha, K. Levin and J. P. Lu, Phys. Rev. 47, 9055 (1993) and references therein.

20. Y. Obata, J. Phys. Soc. Japan 18, 1020 (1963).

21. J. W. Loram et al., Physica C 235-240, 134 (1994).

22. S. M. Hayden et al., Phys. Rev. Lett., 76, 1344 (1996). 


\section{Figure Captions}

Figure 1: Illustrates the physical content of Eq. (10). A Cu d $\mathrm{x}^{2}-\mathrm{y}^{2}$ orbital is surrounded by the $\mathrm{p}_{\mathrm{x}, \mathrm{y}}$ orbitals of oxygen atoms with the phase as shown. A deviation of the phase $\sim \mathbf{q}$ in the direction shown produces a fluctuation with $\mathrm{d}_{\mathrm{xy}}$ symmetry (shown shaded) about the $\mathrm{Cu}$-site leading to a orbital magnetic moment fluctuation $\sim$ q.

Figure 2: Experimental results from Ref. (5) for $\mathrm{Cu}$ nuclear relaxation rate with field the planes in $\mathrm{YBa}_{2} \mathrm{Cu}_{3} \mathrm{O}_{6.9}$, and nuclear quadrupole relaxation rate in $\mathrm{La}_{1.85} \mathrm{Sr}_{.15} \mathrm{CuO}_{4}$ from Ref. (10) compared with the calculations in this paper. The two parameters required are discussed in the text. 